\title{
Quartet structure of self-conjugate nuclei
}

\author{
Michelangelo Sambataro ${ }^{1, *}$ \\ ${ }^{1}$ Istituto Nazionale di Fisica Nucleare - Sezione di Catania, Via S. Sofia 64, I-95123 Catania, Italy
}

\begin{abstract}
We provide a description of even-even $N=Z$ nuclei in a formalism of quartets. Quartets are four-body correlated structures characterized by isospin $T$ and angular momentum $J$. We show that the ground state correlations induced by a realistic shell model interaction can be well accounted for in terms of a restricted set of $T=0$ low- $J$ quartets, the $J=0$ one playing by far a leading role among them. A conceptually similar description of odd-odd self-conjugate nuclei is given in terms of two distinct families of building blocks, one formed by the same $T=0$ quartets employed for the even-even systems and the other by collective pairs with either $T=0$ or $T=1$. Some applications of this formalism are discussed for nuclei in the $s d$ shell.
\end{abstract}

\section{Introduction}

Self-conjugate nuclei are characterized by an equal number of protons and neutrons distributed over the same single particle orbits. In these nuclei, owing to the charge independence of the nuclear interaction, the isovector proton-neutron $(p n)$ pairing force represents an essential component of the effective nuclear interaction. This force acts equally on proton-proton, neutron-neutron as well as $p n$ pairs. In addition, $p n$ pairing is also expected to occur in an isoscalar form. The competition between these two types of $p n$ pairing in $N=Z$ nuclei has been matter of great debate among nuclear physicists [1].

In recent years we have carried out an analysis of the $p n$ pairing in even-even $N=Z$ nuclei both in the isovector and in the isoscalar channels [2-5]. This analysis has evidenced, on the one hand, that a description of the ground state correlations induced by this interaction in terms of a condensate of collective pairs (of various form [5]) is not satisfactory and, on the other hand, that these correlations can be accounted for to a high degree of precision by approximating the ground state as a product of identical $T=0$ quartets. $T=0$ quartets are four-body correlated structures formed by two protons and two neutrons and, in the case of a spherical mean field, they are also characterized by a total angular momentum $J=0$. We have also explored a more sophisticated approximation which consists in letting the quartets to be all distinct and we have verified that it leads to basically exact results in the case of $p n$ isovector pairing in deformed systems [3]. In all cases the quartets have been constructed variationally for each nucleus.

In the presence of a general Hamiltonian, other quartets are reasonably expected to come into play in addition to the $T=0, J=0$ ones emerging from the analysis of the $p n$ pairing. In Ref. [6] we have extended the approach

*e-mail: michelangelo.sambataro@ct.infn.it of Refs. [2-5] to realistic even-even $N=Z$ nuclei and focused our attention not only on the ground state but also on excited states. We have explored the role of quartets of all possible isospins $(T=0,1,2)$ and also of various angular momenta $J$. It has been evidenced that only the $T=0$ quartets play an effective role in the structure of the lowlying states of an even-even $N=Z$ nucleus and, among these, the $J=0$ quartet is by far the most relevant one.

Quartetting is espected to play a key role also in the structure of odd-odd $N=Z$ nuclei. These nuclei exhibit a peculiar coexistence of isospin $T=0$ and $T=1$ states at very low excitation energies. In Ref. [7] we have shown that the basic features of the spectra of these nuclei can be reproduced by resorting to two distinct families of building blocks, one formed by collective $T=0$ quartets and the other by collective pairs with either $T=0$ or $T=1$ isospin. In this contribution we will summarize the basic results that have been achieved for both even-even and odd-odd nuclei.

\section{Quartetting in even-even nuclei}

We work in a spherically symmetric mean field and label the single-particle states by $i \equiv\left\{n_{i}, l_{i}, j_{i}\right\}$, where the standard notation for the orbital quantum numbers is used. The quartet creation operator is defined as

$$
\begin{aligned}
Q_{\alpha, J M, T T_{z}}^{+}= & \sum_{i_{1} j_{1} J_{1} T_{1}} \sum_{i_{2} j_{2} J_{2} T_{2}} C_{i_{1} j_{1} J_{1} T_{1}, i_{2} j_{2} J_{2} T_{2}}^{(\alpha)} \\
& \times\left[\left[a_{i_{1}}^{+} a_{j_{1}}^{+}\right]^{J_{1} T_{1}}\left[a_{i_{2}}^{+} a_{j_{2}}^{+}\right]^{J_{2} T_{2}}\right]_{M T_{z}}^{J T},
\end{aligned}
$$

where $a_{i}^{+}$creates a fermion in the single particle state $i$ and $J(T)$ and $M\left(T_{z}\right)$ denote, respectively, the total angular momentum(isospin) and the relative projections. No restrictions on the intermediate couplings $J_{1} T_{1}$ and $J_{2} T_{2}$ are introduced in the calculations. The criterion adopted for the selection of the quartets has been that of choosing, as representative of the quartets with a given isospin 


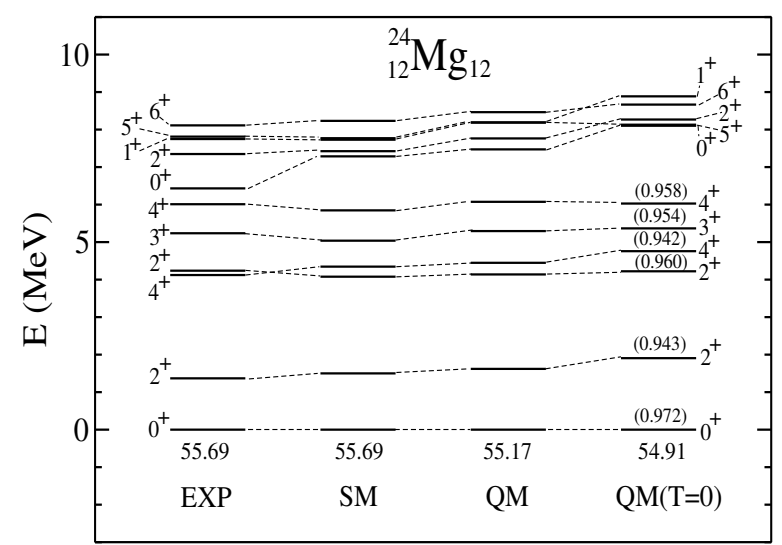

Figure 1. Spectrum of ${ }^{24} \mathrm{Mg}$ obtained in the quartet model (QM) compared to experimental data (EXP) and shell model (SM) results. $\mathrm{QM}(\mathrm{T}=0)$ denotes the results obtained only with $\mathrm{T}=0$ quartets; the numbers on top of these levels are the overlaps between the QM and SM eigenfunctions. The number below each spectrum gives the ground state correlation energy, namely the difference between the total ground state energy and the energy in the absence of interaction.

$T$, those describing the lowest levels with that isospin in nuclei with four active particles outside the inert core of reference [6]. For applications within the $s d$ shell, the inert core is represented by ${ }^{16} \mathrm{O}$ and the nuclei which have therefore been considered for the definition of the quartets are ${ }_{10}^{20} \mathrm{Ne}_{10},{ }_{9}^{20} \mathrm{~F}_{11}$ and ${ }_{8}^{20} \mathrm{O}_{12}$. The lowest states of these nuclei are characterized by $T=0,1$ and 2, respectively. Each of these states therefore identifies a quartet with given $T, J$ (and a projection $T_{z}=(N-Z) / 2$ ). We have performed configuration interaction calculations in spaces built in terms of these selected sets of quartets. We have also explored reductions of these sets to identify the most relevant quartets in the structure of the nuclei under investigation. The USDB interaction [8] has been employed throughout the calculations.

We start our analysis by examining the self-conjugate nucleus ${ }^{24} \mathrm{Mg}$. In Fig. 1, we compare the experimental spectrum of this nucleus with that resulting from a shell model (SM) calculation and with the spectrum obtained in the quartet model (QM) when all the selected $T=0,1,2$ quartets are taken into account. The quartet approach is seen to reproduce well both the SM ground state correlation energy and the SM excited states up to an energy of about $9 \mathrm{MeV}$. The $\mathrm{SM}$ is in turn able to fit well the experimental levels.

Having verified that the selected sets of $T=0,1,2$ quartets are sufficient to describe the low-energy spectrum of ${ }^{24} \mathrm{Mg}$, it becomes of interest to investigate the role of the different quartets. We begin by focusing on isospin. In Fig.

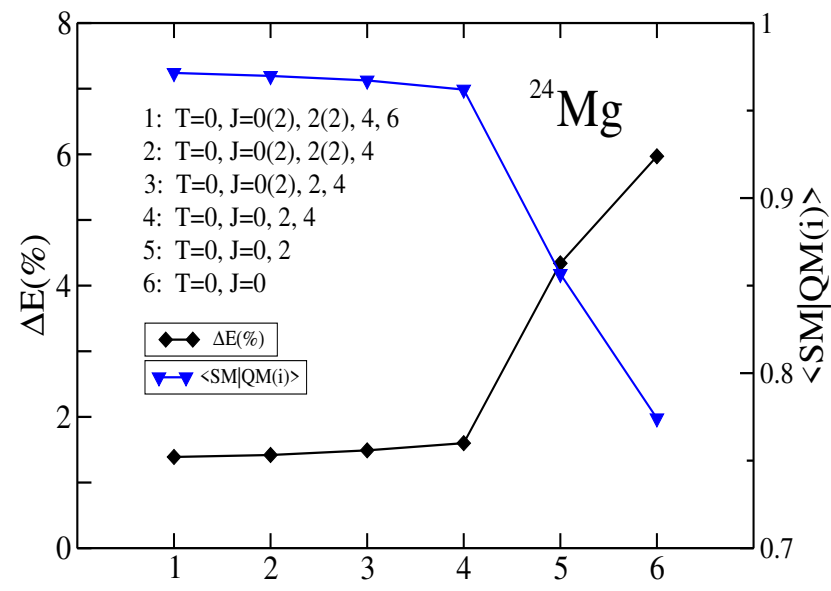

Figure 2. Relative errors (with respect to the SM value) in the ground state correlation energy of ${ }^{24} \mathrm{Mg}$ obtained within the QM with the sets of quartets indicated in the figure. For each set we also show the overlaps between SM and QM wave functions.

1 , on the right hand side, we show the theoretical spectrum obtained in the quartet formalism when only $T=0$ quartets are retained. On top of the lowest levels we also show the overlaps with the corresponding SM eigenstates. One can see that this spectrum does not exhibit relevant differences with respect to the full QM calculation and that the above overlaps are pretty large, what confirms the good quality of the $\mathrm{QM}(T=0)$ wave functions. This result provides a clear evidence of the marginal role played by the $T=1$ and $T=2$ quartets in the structure of these states.

As a next step, we concentrate on the ground state of ${ }^{24} \mathrm{Mg}$ by employing only $T=0$ quartets. In Fig. 2, we show how the error in the correlation energy of this state, relative to the SM result, varies by reducing, one quartet at a time and starting from the highest one in energy, the set of $T=0$ quartets. The correlation energy remains basically unchanged up to the point where only the lowest $J=0,2,4$ quartets are left. From this point on further reductions in the set of quartets lead to significant variations in the energy. The overlaps between the SM and QM ground states which are shown in the same figure exhibit a behavior which is consistent with that of the energies. Thus, these calculations indicate that the $T=0$ quartets with $J=0,2,4$ play a major role in the ground state of ${ }^{24} \mathrm{Mg}$. Among these quartets, the $T=0, J=0$ quartet is by far the one which contributes most to the correlation energy since, as it can be seen in Fig. 2, an approximation in terms of only this quartet accounts for about $94 \%$ of the total energy. A very similar conclusion has emerged also from an analysis of the ground state of the three-quartet system ${ }^{28} \mathrm{Si}[6]$. It is also worthy noticing that the role of the $T=0, J=0$ quartet can be further increased in a sig- 


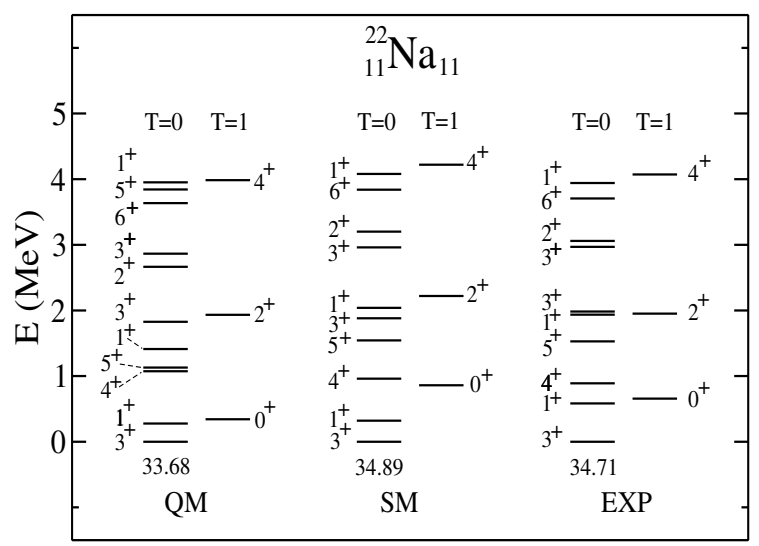

Figure 3. The spectrum of ${ }^{22} \mathrm{Na}$ obtained with the quartet formalism (QM) compared with shell model (SM) and experimental (EXP) spectra. The number below each spectrum is the ground state correlation energy (see Fig. 1).

nificant way if this is constructed variationally for each nucleus [9].

\section{Quartetting in odd-odd nuclei}

The starting point of our analysis of odd-odd $N=Z$ nuclei is the assumption that a state with isospin $T$ can be represented as a superposition of products of one or more $T=0$ quartets and one extra pair with isospin $T$. Thus, within this scheme, the total isospin of the state coincides with that of the pair. Based on the conclusions of the previous analysis on even-even nuclei, we involve in the calculations only three $T=0$ quartets, namely the $J=0,2,4$ quartets describing the lowest three states of ${ }^{20} \mathrm{Ne}$. In analogy, as collective pairs with isospin $T$, we assume those describing the lowest three states with that isospin in ${ }^{18} \mathrm{~F}$ (one proton and one neutron outside the ${ }^{16} \mathrm{O}$ core). These states are characterized by angular momenta $J=1,3,5$ for $T=0$ and $J=0,2,4$ for $T=1$. Once fixed, these quartets and pairs are no longer modified. By defining the creation operator for a collective pair as

$$
P_{J M, T T_{z}}^{+}=\sum_{i j} p_{i j}\left[a_{i}^{+} a_{j}^{+}\right]_{M T_{z}}^{J T},
$$

where $M\left(T_{z}\right)$ stands for the projection of $J(T)$, we perform configuration interaction calculations in the space (we adopt the $m$-scheme)

$$
\left\{Q_{J_{1} M_{1}}^{+} P_{J M, T 0}^{+}|0\rangle\right\}
$$

for ${ }^{22} \mathrm{Na}$ and in the space

$$
\left\{Q_{J_{1} M_{1}}^{+} Q_{J_{2} M_{2}}^{+} P_{J M, T 0}^{+}|0\rangle\right\}
$$

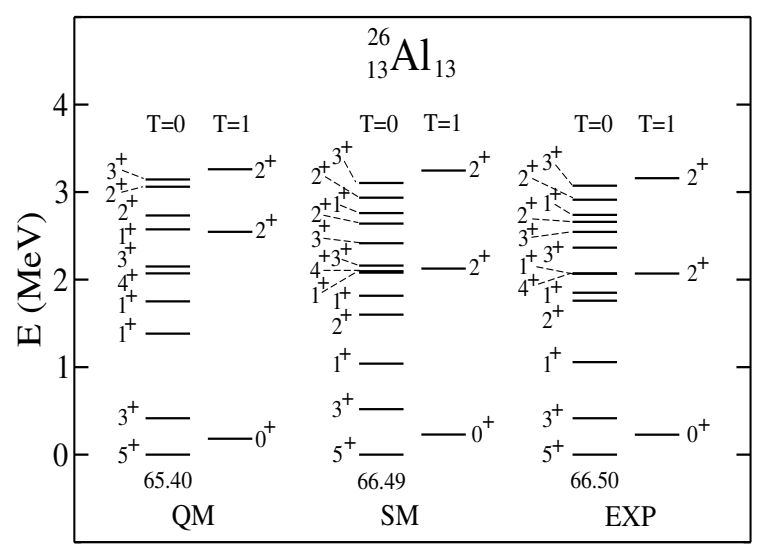

Figure 4. The same as in Fig. 3 for ${ }^{26} \mathrm{Al}$.

for ${ }^{26} \mathrm{Al}$, with $|0\rangle$ representing the reference vacuum.

Fig. 3 illustrates the results obtained in the case of ${ }^{22} \mathrm{Na}$. In this figure we compare the positive-parity experimental spectrum of ${ }^{22} \mathrm{Na}$ up to an energy of about 4 $\mathrm{MeV}$ (EXP) with the spectrum obtained with the present quartet formalism (QM) and with the shell model result (SM). The agreement between QM and SM spectra looks good. This agreement is further emphasized by the values of the overlaps between corresponding QM and SM states. These are: $0.94(J=3), 0.93(J=1), 0.97(J=0), 0.92$ $(J=4), 0.96(J=5)$. In Fig. 4 we show the analogous comparison for ${ }^{26} \mathrm{Al}$. The agreement is satisfactory also in this case although one may notice that three $T=0$ states (with $J=1,2,3$ ) around $2 \mathrm{MeV}$ are missing in the QM spectrum in comparison with the SM one. This suggests that the inclusion of some extra element either in the set of quartets or in that of $T=0$ pairs would be welcome. We have evaluated also in this case the overlaps between the lowest QM and SM eigenstates and found the following values: $0.94(J=5), 0.94(J=0), 0.95(J=3)$, $0.86(J=1)$. These values support the good quality of our approximation scheme.

\section{Conclusions}

We have reported on a description of both even-even and odd-odd $N=Z$ nuclei in the $s d$ shell in a formalism of $T=$ 0 quartets. For all the nuclei that have been analyzed the quartet formalism has provided a description of the lowenergy spectra comparable in accuracy with that of shell model calculations. This fact confirms the importance of quartet degrees of freedom in $N=Z$ nuclei and validates the present quartet formalism as the appropriate tool for treating them. 


\section{References}

[1] S. Frauendorf and A.O. Macchiavelli, Progr. Part. Nucl. Phys. 78, 24 (2014)

[2] N. Sandulescu, D. Negrea, J. Dukelsky, and C.W. Johnson, Phys. Rev. C 85, 061303(R) (2012)

[3] M. Sambataro and N. Sandulescu, Phys. Rev. C 88, 061303(R) (2013)

[4] M. Sambataro, N. Sandulescu, and C.W. Johnson, Phys. Lett. B 740, 137 (2015)
[5] M. Sambataro and N. Sandulescu, Phys. Rev. C 93, 054320 (2016)

[6] M. Sambataro and N. Sandulescu, Phys. Rev. Lett. 115, 112501 (2015)

[7] M. Sambataro and N. Sandulescu, Phys. Lett. B 763, 151 (2016)

[8] B.A. Brown and W.A. Richter, Phys. Rev. C 74, 034315 (2006)

[9] M. Sambataro and N. Sandulescu, J. Phys. G: Nucl. Phys. 40, 055107 (2013) 